Originally published as:

Pablo E. Brockmann, Michael S. Urschitz, Martin Schlaud, Christian F. Poets.

Primary snoring in school children: prevalence and neurocognitive impairments. (2012) Sleep and Breathing, 16, pp. 23-29.

DOI: $10.1007 / \mathrm{s} 11325-011-0480-6$

This is an author manuscript.

The definitive version is available at: http://link.springer.com 


\title{
Primary snoring in school children: prevalence and neurocognitive impairments
}

\author{
Pablo E. Brockmann ${ }^{1,2}$, Michael S. Urschitz ${ }^{1}$, Martin Schlaud ${ }^{3}$, Christian F. Poets ${ }^{1}$ \\ P. Brockmann and M. Urschitz contributed equally to this publication. \\ ${ }^{1}$ Working Group on Paediatric Sleep Medicine, Department of Neonatology, University Children's \\ Hospital, University of Tuebingen, Tuebingen, Germany \\ ${ }^{2}$ Department of Paediatrics, Universidad Catolica de Chile, Santiago, Chile \\ ${ }^{3}$ Department of Epidemiology and Health Reporting, Robert Koch Institute, Berlin, Germany
}

\begin{abstract}
Purpose: We aimed to investigate the prevalence of primary snoring (PS) and its association with neurocognitive impairments.

Methods: Data from a community-based study in 1,114 primary school children were used to identify children who never ( $N=410)$ or habitually snored $(N=114)$.

In order to separate children with PS from those with upper airway resistance syndrome (UARS) or obstructive sleep apnoea (OSA), home polysomnography was conducted in all habitually snoring children. Neurocognitive impairments and poor school performance were compared between children who never snored, PS, and UARS/OSA
\end{abstract}

Results: Polysomnography was successfully conducted in 92 habitual snorers. Of these, 69 and 23 had PS and UARS/ OSA, respectively. Prevalence [95\% confidence interval (95\% CI)] of PS was $6.1 \%$ (4.5-7.7).

Compared to children who had never snored, children with PS had more hyperactive (39\% vs. 20\%) and inattentive behavior (33\% vs. $11 \%)$, as well as poor school performance in mathematics $(29 \%$ vs. $16 \%)$, science (23\% vs. $12 \%)$, and spelling (33\% vs. $20 \%$; all $P$ values $<0.05)$. PS was a significant risk factor (odds ratio; $95 \% \mathrm{Cl}$ ) for hyperactive behaviour (2.8; 1.6-4.8), inattentive behaviour (4.4; 2.4-8.1), as well as daytime sleepiness (10.7; 4.0-28.4).

PS was also an independent risk factor for poor school performance in mathematics (2.6; 1.2-5.8), science $(3.3 ; 1.2-8.8)$, and spelling $(2.5 ; 1.1-5.5)$. Odds ratios throughout were similar to the UARS/OSA group.

Conclusions: Children with non-hypoxic, non-apnoeic PS may exhibit significant neurocognitive impairments. Consequences may be similar to those associated with UARS or OSA. If confirmed, PS is not "benign" and may require treatment. 


\section{Introduction}

Primary snoring (PS) and obstructive sleep apnoea (OSA) are considered to be expressions of sleepdisordered breathing (SDB) in children [1]. Based on standard polysomnographic definitions [2, 3], some authors have postulated that PS is at the lower end of the SDB severity spectrum where treatment is routinely not considered necessary [3].

However, there is increasing evidence that neurocognitive impairments $(\mathrm{NCl})$ are more frequent in children with PS compared to children who have never snored.

In these studies, PS was associated with several cognitive disabilities [4], problems in memory [5], language and visuospatial areas [6], and poor academic performance [7].

Underlying mechanisms that may explain the association between PS and the above-mentioned consequences are not well understood.

While intermittent hypoxia may be an important factor in children with OSA [8, 9], hypoxia is not an explanation applicable to children with PS. Furthermore,

poor academic performance has also been found in children with SDB without intermittent hypoxia [10, 11]. Other possible mechanisms that may be involved are arousal, sleep fragmentation, and sleep disruption [12, 13]. Previous studies, however, may not have clearly separated PS from other forms of SDB like the upper airway resistance syndrome (UARS) $[5,11,14]$.

Hence, worse outcome related to PS may in fact have been related to UARS. Embedded in our German study on SDB in primary school children [15], we aimed to (1) separate UARS and OSA from PS, (2) estimate the prevalence of PS, and (3) elucidate if PS is associated with NCl-like behavioural problems or poor academic performance.

\section{Methods}

\section{Subjects}

Recruitment strategy, basic characteristics of the underlying source population and study sample, as well as analyses for representativeness are given elsewhere [15].

In short, 27 of the 59 public primary schools located within the city limits of Hannover, Germany, were randomly selected.

After approval by the institutional review board (i.e. Ethik Kommission der Medizinischen Hochschule, Hannover, Germany; study approval number 2,494) and the regional directorate of education, all children attending third-grade classes in these schools $(\mathrm{N}=1,760)$ were identified and contacted in their classrooms.

A cover letter explaining the study and an informed consent form were given to the children to be filled out by their parents. Finally, 1,144 children $(65.0 \%$ of all contacted third graders) participated in the study. Comparisons to all contacted third graders $(\mathrm{N}=1,760)$ and the underlying source population of third graders $(\mathrm{N}=3,809)$ revealed good to excellent representativeness concerning gender distribution, socioeconomic status, academic performance, and diagnosis of asthma. Race was not recorded, as the prevalence estimation of non-Caucasians among participants was less than $5 \%$.

SDB symptoms were assessed by the OSA score [16]. Snoring was investigated with the question "Does your child snore?". Children were classified as habitual snorers if the answers were "frequently" or "always" and if the answer was "never", as never snorers. Parental education was investigated separately for either parent. The highest graduation from school (four-point rating scale: "no graduation/primary school", "secondary school", "high school", and "college/university") was scored. Low parental education was defined as "no graduation/primary school". Body mass index was calculated using a standard formula: weight $(\mathrm{kg}) /[$ height $(\mathrm{m}) \times$ height $(\mathrm{m})]$. 


\section{Polysomnography}

Habitual snorers were contacted by telephone and invited to participate in a nocturnal home sleep study using a portable device (Embletta PDS; Embla, Broomfield, CO, USA).

A detailed description of this method is presented elsewhere [17]. Briefly, recordings were done overnight at the children's homes.

Parents were instructed how to handle the device. Polysomnographic montage comprised chest and abdominal wall movements (piezo sensors; Pro-Tech, USA); nasal pressure and linearized nasal airflow estimation (nasal prongs and built-in pressure transducer; Embla, USA); oral airflow (thermocouple; Pro-Tech); snoring (vibration sensor; New Life Technologies, USA); arterial haemoglobin oxygen saturation measured by pulse oximetry, pulse rate, and pulse waveform (pulse oximeter Xpod; Nonin Medical, USA); actigraphy and body position (builtin actimeter/body position sensor; Embla, USA); and user events (built-in event button; Embla, USA).

Recordings were analyzed using device-specific software (Somnologica for Embletta 3.3; Embla, USA). Sleep onset was estimated as the beginning of the first 10 -min period not containing any changes in body position, morning awakening as the end of the last such 10-min period [17]. Estimated sleep time was calculated as the time between sleep onset and morning awakening. Recordings were analyzed for artefactual or uninterpretable periods on the nasal flow, thoracic effort, abdominal effort, or oximetry channel.

Movement periods and artefactual/uninterpretable periods were excluded from estimated sleep time if they lasted for more than $5 \mathrm{~min}$, and the corrected estimated sleep time was calculated [17].

A minimum of $4 \mathrm{~h}$ of corrected estimated sleep time was required.

Respiratory events were analyzed manually according to current guidelines [18]. A central apnoea was scored if criteria for apnoea were fulfilled and no chest and abdominal wall movements were present. An obstructive apnoea was scored if criteria for apnoea were fulfilled and out-of-phase movements of the chest and abdomen were present.

Mixed apnoeas were defined as apnoeas with central and obstructive components. A hypopnoea was scored if the amplitude of the nasal airflow fell to $<50 \%$ of the average amplitude of the two preceding breaths, arterial oxygen desaturation of at least three percentage points occurred within $30 \mathrm{~s}$ of the onset of the event, and the event comprised at least two breath cycles.

Flow limitations (i.e. reduction of the nasal airflow amplitude by $>50 \%$ for more than two breathing cycles, not associated with an arterial oxygen desaturation) were also scored. An arterial oxygen

desaturation was defined as a fall in arterial oxygen saturation of at least three percentage points. The apnoea-hypopnoea index $(\mathrm{AHI})$ was calculated as the number of obstructive and mixed apnoeas and hypopnoeas, divided by corrected estimated sleep time.

The respiratory disturbance index (RDI) was calculated as the number of obstructive and mixed apnoeas, hypopnoeas, and flow limitations, divided by corrected estimated sleep time. The oxygen desaturation index was calculated as the number of desaturation events divided by corrected estimated sleep time.

According to current clinical guidelines, OSA was defined as $A H I \geq 1$ [19]. In the absence of generally accepted definitions for UARS and PS, we defined UARS as AHI $<1$ and RDI $\geq 1$, and PS as AHI as well as RDI $<1$ and oxygen desaturation index $<4$ [17, 20-22].

\section{Daytime symptoms}

Hyperactive-inattentive behaviour was investigated with the questions "Is your child very restless, fidgety, or always in motion during daytime?" and "Does your child have difficulties concentrating during daytime?".

Daytime sleepiness was investigated with the questions "Is your child tired during daytime?", "Does your child fall asleep while watching TV?", and "Does your child fall asleep at school?".

Answers were rated on a five- point rating scale (never, rarely, occasionally, frequently, and almost always).

For calculation of a hyperactivity-inattention score (range 0-4) and an excessive daytime sleepiness score (range 0-6), arbitrary numerical scores were assigned to each of the above-mentioned answers ranging from 0 (never or rarely) to 1 (occasionally) and 2 (frequently or almost always). 
Regarding the questions on hyperactive-inattentive behaviourand one question on daytime sleepiness (i.e. "tired during daytime"), the categories "frequently" and "almost always" were collapsed into "problem present"; the remaining categories were collapsed into "problem absent".

Regarding the other questions on daytime sleepiness, "never" was categorized as "problem absent"; the remaining categories were collapsed into "problem present".

\section{School performance}

With parental consent, last term's report form was obtained from the school archive. This form included written ratings on a six-point scale ( 1 for "outstanding" and 6 for "failed")

for mathematics, science, and spelling.

These school subjects were chosen because they had shown to correlate with habitual snoring [11].

Poor academic performance was defined as grade 4-6 on the scale (i.e. approximately the

lowest quintile grades) [11].

In order to compare each subject to his/her classroom peers, the frequency of all

grades were provided for each class and school subject by the class teachers. Using this class-related

frequency distribution, individual grades were transformed into class and school subject stratified

performance percentiles [e.g. the best grade within one class/school subject corresponded

to $100 \%$ performance; a performance of $80 \%$ meant that only $20 \%$ of peers performed better (i.e. had a higher grade), etc.]

\section{Statistical analysis}

This study had a complex sampling procedure comprising two stages, stratification, and cluster sampling [15, 23]. The two stages were: (1) first stage, schools were selected from a list of all eligible schools and (2) second stage, individuals were sampled from selected schools (i.e. multistage sampling).

Prior to sampling, schools were divided into three different strata based on the socioeconomic status of the schools (i.e. stratified sampling).

The primary sampling unit was a school while the unit of concern was an individual pupil within that school. The sampling variation for a given number of individuals is smaller for cluster sampling than for simple random sampling [24].

This has to be taken into account in the analysis. In addition, unbalanced selection and response proportions within strata and clusters had occurred [23].

That means that the probability for individuals being in the final study sample was different among strata and clusters.

These different probabilities of selection have to be taken into account, too, in order to obtain a largely unbiased point estimate of the population prevalence of PS. Therefore, sampling weights were calculated as the inverse of the overall selection probability and used for adjusting the point estimate [24]. This value reflects the number of pupils that each of the sampled individuals represent.

To cope with the complex sampling procedure, the survey module provided by Stata 9.2 (Stata Corp., College Station, TX, USA) was used for estimating the design-adjusted point estimate for the population prevalence of PS and its $95 \%$ confidence interval $(95 \% \mathrm{Cl})$.

All other analyses were done with PASW Statistics 18

(IBM SPSS Statistics 18, Chicago, IL). Descriptive statistics were used to summarize subject characteristics, results of questions on SDB and daytime symptoms, and polysomnography.

Outcomes (i.e. daytime symptoms and school performance) were compared with one-way analysis of variance between the three SDB categories: neversnoring (i.e. reference group), PS, and UARS/OSA.

In order to investigate potential associations between PS and daytime symptoms or poor school performance, odds ratios (OR) and their $95 \% \mathrm{Cls}$ were calculated using unconditional logistic regression. Logistic models were adjusted for gender and age (continuous variable) for the analysis of associations with daytime symptoms. For analysis of associations with poor school performance, logistic models were additionally adjusted for maternal and paternal education (two 
categorical variables). To adjust for different rating styles of teachers, class membership was entered as a dummy variable. To enable a complete data set, missing values within confounding variables were replaced by the same value to form a distinct "missing" category. This category was included in each regression analysis. $P$ values $<0.05$ were considered statistically significant.

\section{Results}

Of 1,144 children included, $410(36 \%)$ were classified as never snorers and $114(10 \%)$ as habitual snorers. Of the latter, 16 did not participate in polysomnography.

Of 98 conducted polysomnographies, 6 were excluded from analysis [i.e. 2 uninterpretable recordings, 3 had a corrected estimated sleep time $<4 \mathrm{~h}$, and 1 showed an increased AHI (i.e. 5.3) exclusively due to central apnoeas]. Figure 1 shows the sampling results.

Finally, 69 habitual snorers were classified as PS, the remaining 11 as UARS, and 12 as OSA. Demographic data are presented in Table 1. In contrast to age and gender distribution, body mass index increased and maternal education decreased with SDB severity.

The designadjusted point estimate $(95 \% \mathrm{Cl})$ for the prevalence of PS was $6.1 \%(4.5-7.7)$.

Results for daytime symptoms and school performance are given in Table 2, and regressions adjusted for possible confounders in Table 3. Hyperactive and inattentive behaviour scores, daytime sleepiness scores, and school performance percentiles were significantly different between never snoring, PS, and UARS/OSA. Hyperactive and inattentive behaviour as well as daytime sleepiness were more frequently reported in children with PS compared to never snorers.

These associations remained significant in multiple logistic regression analysis after adjusting for age and gender. For the UARS/OSA group, ORs were above 1 throughout, although significant only for inattentive behavior and falling asleep while watching TV.

In addition, both groups, PS and UARS/OSA, performed poorer at school. Compared to children who never snored,

ORs were significantly $>1$ for all three school subjects under study in the PS group. In the UARS/OSA group, however, ORs were only significantly higher for poor school performance in science. Also here, these associations remained significant after adjusting for age, gender, and parental education.

\section{Discussion}

In our study, PS was common and associated with significant $\mathrm{NCl}$ in primary school children. Even after adjusting for potential confounders, children with PS had a higher risk for hyperactive and inattentive behaviour, daytime sleepiness, and poor school performance compared to children who never snored. To our knowledge, this is the first study that assessed the prevalence of PS in primary school children. Even after careful separation from other forms of SDB, associations between PS and $\mathrm{NCl}$ remained significant and relevant.

Furthermore, children with PS presented with similarly increased risks for $\mathrm{NCl}$ compared to those in the UARS/OSA group.

Studies that investigated the association between PS and $\mathrm{NCl}$ are sparse $[6,25,26]$. Blunden at al. showed significantly impaired attention, lower memory and intelligence scores in 16 children with PS or "mild" OSA, compared to 16 children who had never snored [5].

In the same subjects, Kennedy et al. showed a significant relationship between oxygen desaturations, obstructive hypopnoeas, and respiratory arousals on one hand and $\mathrm{NCl}$ on the other [4]. O'Brien et al. demonstrated also several $\mathrm{NCl}$ in children with PS [6].

In that study, children with PS showed significantly more respiratory arousals than non-snoring children [6]. Hence, the presence of respiratory events and/or respiratory-related arousal may have confounded the relationship with $\mathrm{NCl}$ in thesestudies. In fact, associations may have been related to UARS, not to PS.

Additional evidence for a causal relationship between PS and $\mathrm{NCl}$ came from studies that assessed the impact of treatment (e.g. adenotonsillectomy) on $\mathrm{NCl}$. In one study, aggressive, inattentive, and 
hyperactive behaviour significantly decreased following treatment [27].

Nevertheless, SDB was detected using home video and pulse oximetry and no polysomnography was conducted in that study.

In another study, hyperactive behaviour and attention improved

after adenotonsillectomy in children with PS [28]. Of interest, the improvement was similar in subjects with PS compared to those with OSA.

Mechanism(s) for the link between PS and $\mathrm{NCl}$ still remain unclear. It is thought that intermittent hypoxia, sleep fragmentation, and sleep disruption are physiological correlates of SDB, suspected to cause prefrontal cortical dysfunction leading to impaired cognitive execution [9].

Nevertheless, intermittent hypoxia is not applicable to PS, which does not present with hypoxia or apnoea.

The other postulated mechanisms (i.e. sleep fragmentation and disruption) could explain why children with PS show significant $\mathrm{NCl}$ even in the absence of hypoxia [12, 29]. Notwithstanding this, previous studies have failed to demonstrate differences in electroencephalographic arousals between children with PS and children who have never snored [30]. However, other assessments of sleep fragmentation and disruption like (1) sleep pressure score [31]; (2) cycling alternating patterns [32]; or (3) subcortical arousal detected by beat-to-beat blood pressure [33], heart rate [34, 35], and pulse transit time [36-38] may help better to understand underlying mechanisms.

The lack of a diagnostic marker for the identification of children with PS at risk for $\mathrm{NCl}$ is of special concern. Future studies addressing the predictive value of standard and/or new polysomnographic parameters and thresholds seem therefore necessary.

On the other hand, associations between PS and $\mathrm{NCl}$ could reflect a variety of social and health factors. Although we adjusted for important confounders such as age, gender, or parental education, some residual confounding might have remained unconsidered. As shown by Chervin et al., poor school performance in primary school children was independently predicted by low socioeconomic level but not by symptoms of SDB [39]. In another study, snoring was independently associated with socioeconomic deprivation[40].

In our study, we also found an increased frequency of low maternal education and higher body mass indices in children with PS and UARS/OSA compared to those who never snored. However, the precise relationship between PS, low socioeconomic level, obesity, and $\mathrm{NCl}$ remains to be clarified.

Some limitations of the study have been discussed. In short, electroencephalography, electrooculography, and electromyography were not part of the ambulatory polysomnographic setting. We were, hence, unable to differentiate between wakefulness and sleep, between different sleep stages, or between cortical and autonomic arousal.

Nevertheless, previous studies showed that abbreviated home polysomnography has good to excellent correlation regarding respiratory events and indices when compared to full sleep lab-based polysomnography [41, 42].

Therefore, we postulate that use of this abbreviated technique in a large community setting is able to differentiate OSA and UARS from PS based on respiratory indices.

We tried to thoroughly separate OSA and UARS from PS using respiratory events including flow limitations. However, it is possible that some children with subtle forms of UARS were still misclassified as PS because we did not use oesophageal pressure monitoring, the gold standard for detecting UARS [43], and electroencephalography, the gold standard for assessing sleep fragmentation by multiple cortical arousal [44]. Use of oesophageal pressure monitoring, however, did not improve detection of UARS if a nasal pressure transducer was used [28].

Moreover, nasal and oesophageal pressure measurements correlated well and the presence of flow limitation indicated airway resistance measured by oesophageal pressure monitoring in a validation study [45]. We, hence, believe that the use of a nasal pressure transducer, which allowed detection of flow limitations, enabled us to identify children with probable UARS and, thereby, to separate them from children with PS.

Nevertheless, misclassification of some subjects with PS cannot be fully excluded. Another limitation is the fact that daytime behavioural assessment was based solely on parental questionnaires and not on formal ratings by independent and blinded investigators. 
This might have introduced bias and overestimated the association between PS and $\mathrm{NCl}$.

\section{Conclusion}

Children with PS may have significant $\mathrm{NCI}$ that are similar to those associated with UARS/OSA

Considering the high prevalence of PS in childhood, identification of children susceptible to NCI may be of major health relevance as they might benefit from early treatment. 


\section{References}

1. Marcus CL (2001) Sleep-disordered breathing in children. Am J Respir Crit Care Med 164:16-30

2. American Thoracic Society (1996) Standards and indications for cardiopulmonary sleep studies in children. Am J Respir Crit Care Med 153:866-878

3. American Academy of Pediatrics, Section on Pediatric Pulmonology, Subcommittee on Obstructive Sleep Apnea Syndrome (2002) Clinical practice guideline: diagnosis and management of childhood obstructive sleep apnea syndrome. Pediatrics 109:704-712

4. Kennedy JD, Blunden S, Hirte C, Parsons DW, Martin AJ, Crowe E, Williams D, Pamula Y, Lushington K (2004) Reduced neurocognition in children who snore. Pediatr Pulmonol 37:330-337

5. Blunden S, Lushington K, Kennedy D, Martin J, Dawson D (2000) Behavior and neurocognitive performance in children aged 5-10 years who snore compared to controls. J Clin Exp Neuropsychol 22:554-568

6. O'Brien LM, Mervis CB, Holbrook CR, Bruner JL, Klaus CJ, Rutherford J, Raffield TJ, Gozal D (2004) Neurobehavioral implications of habitual snoring in children. Pediatrics 114:44-49

7. Urschitz MS, Eitner S, Guenther A, Eggebrecht E, Wolff J, Urschitz-Duprat PM, Schlaud M, Poets CF (2004) Habitual snoring, intermittent hypoxia, and impaired behavior in primary school children. Pediatrics 114:1041-1048

8. Gozal D, Daniel JM, Dohanich GP (2001) Behavioral and anatomical correlates of chronic episodic hypoxia during sleep in the rat. J Neurosci 21:2442-2450

9. O'Brien LM, Gozal D (2002) Behavioural and neurocognitive implications of snoring and obstructive sleep apnoea in children: facts and theory. Paediatr Respir Rev 3:3-9

10. Gottlieb DJ, Yao Q, Redline S, Ali T, Mahowald MW (2000) Does snoring predict sleepiness independently of apnea and hypopnea frequency? Am J Respir Crit Care Med 162:1512-1517

11. Urschitz MS, Guenther A, Eggebrecht E, Wolff J, Urschitz-Duprat PM, Schlaud M, Poets CF (2003) Snoring, intermittent hypoxia and academic performance in primary school children. Am J Respir Crit Care Med 168:464-468

12. Montgomery-Downs HE, Gozal D (2006) Snore-associated sleep fragmentation in infancy: mental development effects and contribution of secondhand cigarette smoke exposure. Pediatrics 117: e496-e502

13. Beebe DW, Gozal D (2002) Obstructive sleep apnea and the prefrontal cortex: towards a comprehensive model linking nocturnal upper airway obstruction to daytime cognitive and behavioral deficits. J Sleep Res 11:1-16

14. Blunden S, Lushington K, Lorenzen B, Martin J, Kennedy D (2005) Neuropsychological and psychosocial function in children with a history of snoring or behavioral sleep problems. J Pediatr 146:780-786

15. Schlaud M, Urschitz MS, Urschitz-Duprat PM, Poets CF (2004) The German study on sleepdisordered breathing in primary school children: epidemiological approach, representativeness of study sample, and preliminary screening results. Paediatr Perinat Epidemiol 18:431-440

16. Brouilette R, Hanson D, David R, Klemka L, Szatkowski A, Fernbach S, Hunt C (1984) A diagnostic approach to suspected obstructive sleep apnea in children. J Pediatr 105:10-14

17. Moss D, Urschitz MS, von Bodman A, Eitner S, Noehren A, Urschitz-Duprat PM, Schlaud M, Poets CF (2005) Reference values for nocturnal home polysomnography in primary schoolchildren.

Pediatr Res 58:958-965 
18. Iber C, Ancoli-Israel S, Chesson A, Quan SF, for the American Academy of Sleep Medicine (2007) The AASM manual for the scoring of sleep and associated event: rules, terminology and technical specifications, 1st edn. American Academy of Sleep Medicine, Westchester

19. American Academy of Sleep Medicine (2006) International classification of sleep disorders, 2nd edn. American Academy of Sleep Medicine, Westchester

20. Urschitz MS, Wolff J, Von Einem V, Urschitz-Duprat PM, Schlaud M, Poets CF (2003) Reference values for nocturnal home pulse oximetry during sleep in primary school children. Chest 123:96-101

21. Uliel S, Tauman R, Greenfeld M, Sivan $Y$ (2004) Normal polysomnographic respiratory values in children and adolescents. Chest 125:872-878

22. Verhulst SL, Schrauwen N, Haentjens D, Van Gaal L, De Backer WA, Desager KN (2007) Reference values for sleep-related respiratory variables in asymptomatic European children and adolescents. Pediatr Pulmonol 42:159-167

23. Urschitz MS, Brockmann PE, Schlaud M, Poets CF (2010) Population prevalence of obstructive sleep apnoea in a community of German third graders. Eur Respir J 36:556-568

24. Dohoo IR, Martin W, Stryhn H (2003) Veterinary epidemiologic research. AVC Inc., Charlottetown

25. Rosen CL, Storfer-Isser A, Taylor HG, Kirchner HL, Emancipator JL, Redline S (2004) Increased behavioral morbidity in school-aged children with sleep-disordered breathing. Pediatrics 114:1640 1648

26. Beebe DW, Wells CT, Jeffries J, Chini B, Kalra M, Amin R (2004) Neuropsychological effects of pediatric obstructive sleep apnea. J Int Neuropsychol Soc 10:962-975

27. Ali NJ, Pitson D, Stradling JR (1996) Sleep disordered breathing: effects of adenotonsillectomy on behaviour and psychological functioning. Eur J Pediatr 155:56-62

28. Chervin RD, Ruzicka DL, Giordani BJ, Weatherly RA, Dillon JE, Hodges EK, Marcus CL, Guire KE (2006) Sleep-disordered breathing, behavior, and cognition in children before and after adenotonsillectomy. Pediatrics 117:e769-e778

29. Blunden SL, Beebe DW (2006) The contribution of intermittent hypoxia, sleep debt and sleep disruption to daytime performance deficits in children: consideration of respiratory and nonrespiratory sleep disorders. Sleep Med Rev 10:109-118

30. O'Brien LM, Holbrook CR, Mervis CB, Klaus CJ, Bruner JL, Raffield TJ, Rutherford J, Mehl RC, Wang M, Tuell A, Hume BC, Gozal D (2003) Sleep and neurobehavioral characteristics of 5- to 7-year-old children with parentally reported symptoms of attention-deficit/hyperactivity disorder. Pediatrics 111:554-563

31. Tauman R, O'Brien LM, Holbrook CR, Gozal D (2004) Sleep pressure score: a new index of sleep disruption in snoring children. Sleep 27:274-278

32. Lopes MC, Guilleminault C (2006) Chronic snoring and sleep in children: a demonstration of sleep disruption. Pediatrics 118: e741-e746

33. Tauman R, O'Brien LM, Mast BT, Holbrook CR, Gozal D (2004) Peripheral arterial tonometry events and electroencephalographic arousals in children. Sleep 27:502-506

34. Brockmann PE, Urschitz MS, Noehren A, Sokollik C, Schlaud M, Poets CF (2011) Risk factors and consequences of excessive autonomic activation during sleep in children. Sleep Breath. doi:10.1007/s11325-010-0349-0. published online 18 April 2010

35. Aljadeff G, Gozal D, Schechtman VL, Burrell B, Harper RM, Ward SL (1997) Heart rate variability in children with obstructive sleep apnea. Sleep 20:151-157 
36. Brietzke SE, Katz ES, Roberson DW (2007) Pulse transit time as a screening test for pediatric sleep-related breathing disorders. Arch Otolaryngol Head Neck Surg 133:980-984

37. Katz ES, Lutz J, Black C, Marcus CL (2003) Pulse transit time as a measure of arousal and respiratory effort in children with sleepdisordered breathing. Pediatr Res 53:580-588

38. Rizzoli A, Urschitz MS, Sautermeister J, Miano S, Pagani J, VillaMP, Poets CF (2009) Pulse transit time for scoring subcortical arousal in infants with obstructive sleep apnea. Sleep Breath 13:137-146

39. Chervin RD, Clarke DF, Huffman JL, Szymanski E, Ruzicka DL, Miller V, Nettles AL, Sowers MR, Giordani BJ (2003) School performance, race, and other correlates of sleep-disordered breathing in children. Sleep Med 4:21-27

40. Kuehni CE, Strippoli MP, Chauliac ES, Silverman M (2008) Snoring in preschool children: prevalence, severity and risk factors. Eur Respir J 31:326-333

41. Jacob SV, Morielli A, Mograss MA, Ducharme FM, Schloss MD, Brouillette RT (1995) Home testing for pediatric obstructive sleep apnea syndrome secondary to adenotonsillar hypertrophy. Pediatr Pulmonol 20:241-252

42. Alonso Alvarez ML, Teran Santos J, Cordero Guevara JA, Navazo Eguia Al, Ordax Carbajo E, Masa Jimenez JF, Pelayo R (2008) Reliability of respiratory polygraphy for the diagnosis of sleep apnea-hypopnea syndrome in children. Arch Bronconeumol 44:318-323

43. Guilleminault C, Stoohs R, Clerk A, Cetel M, Maistros P (1993) A cause of excessive daytime sleepiness. The upper airway resistance syndrome. Chest 104:781-787

44. Bonnet M, Carley DW, Carskadon MA, Easton P, Guilleminault C, Harper R, Hayes B, Hirshkowitz M, Ktonas PY, Keenan S, Pressman M, Roehrs T, Smith J, Walsh JK, Weber S, Westbrook PR (1992) EEG arousals: scoring rules and examples: a preliminary report from the Sleep Disorders Atlas Task Force of the American Sleep Disorders Association. Sleep 15:173-84

45. Trang H, Leske V, Gaultier C (2002) Use of nasal cannula for detecting sleep apneas and hypopneas in infants and children. Am J Respir Crit Care Med 166:464-468 


\section{Tables and Figures}

Table 1 Comparisons between SDB categories

SDB sleep disordered breathing, UARS upper airway resistance syndrome, OSA obstructive sleep apnea, SD standard deviation

Table 2 Daytime symptoms and school performance, stratified by SDB category

Characteristic

Never snoring $N=410$ mean (SD)

Hyperactivity-inattention score ${ }^{\mathrm{a}}$

$1.5(1.2)$

$0.6(0.6)$

$70.8(28.9)$

$71.6(27.8)$

$72.6(26.1)$

Excessive daytime sleepiness score ${ }^{\mathrm{a}}$

School performance percentile in mathematics

School performance percentile in science
Primary snoring $N=69$ mean (SD)

$2.4(1.2)$
$1.3(0.9)$
$58.8(30.7)$
$64.3(29.2)$
$66.5(29.3)$

$66.5(29.3)$
UARS/OSA $N=23$ mean (SD)

$2.0(1.2)$

$<0.0001$

$P$ values obtained using one-way analysis of variance

SDB sleep disordered breathing, SD standard deviation, UARS upper airway resistance syndrome, OSA obstructive sleep apnea,

${ }^{a}$ Arbitrary numerical scores were assigned to answers ranging from 0 (never or rarely) to 1 (occasionally) and 2 (frequently or almost always) 
Table 3 Prevalence and adjusted odds ratios for daytime symptoms, stratified by SDB category

\begin{tabular}{|c|c|c|c|c|c|}
\hline \multirow[t]{2}{*}{ Characteristic } & \multirow{2}{*}{$\frac{\text { Never snoring } N=410}{N(\%)}$} & \multicolumn{2}{|c|}{ Primary snoring $N=69$} & \multicolumn{2}{|c|}{ UARS/OSA $N=23$} \\
\hline & & $N(\%)$ & OR $(95 \% \mathrm{Cl})$ & $N(\%)$ & OR $(95 \% \mathrm{CI})$ \\
\hline Hyperactive behaviour $^{\mathrm{a}}$ & $82(20)$ & $27(39)$ & $2.8(1.6-4.8)$ & $7(30)$ & $1.7(0.7-4.4)$ \\
\hline Inattentive behaviour $^{\mathrm{a}}$ & $45(11)$ & $23(33)$ & $4.4(2.4-8.1)$ & $7(30)$ & $3.8(1.4-9.9)$ \\
\hline Tired during daytime ${ }^{\mathrm{a}}$ & $8(2)$ & $11(16)$ & $10.7(4.0-28.4)$ & $2(9)$ & $4.7(0.9-23.9)$ \\
\hline Falling asleep while watching $\mathrm{TV}^{\mathrm{b}}$ & $100(24)$ & $30(44)$ & $2.6(1.5-4.4)$ & $11(48)$ & $2.8(1.2-6.6)$ \\
\hline Falling asleep at school ${ }^{\mathrm{b}}$ & $5(1)$ & $5(7)$ & $6.9(1.9-24.8)$ & $0(0)$ & - \\
\hline Poor school performance in mathematics & $66(16)$ & $20(29)$ & $2.6(1.2-5.8)$ & $8(35)$ & $2.6(0.8-8.7)$ \\
\hline Poor school performance in science & $51(12)$ & $16(23)$ & $3.3(1.2-8.8)$ & $7(30)$ & $5.0(1.04-24.0)$ \\
\hline Poor school performance in spelling & $81(20)$ & $23(33)$ & $2.5(1.1-5.5)$ & $7(30)$ & $0.9(0.3-3.3)$ \\
\hline
\end{tabular}

Statistically significant outcomes are typed in bold. Reference category is "never snoring". All ORs are adjusted for gender and age. ORs for poor school performance in mathematics, science, and spelling are additionally adjusted for paternal and maternal education and class membership SDB sleep disordered breathing, $\mathrm{Cl}$ confidence interval, OSA obstructive sleep apnea, OR odds ratio, UARS upper airway resistance syndrome

a Response categories "frequently" and "almost always" were collapsed into "problem present"; the remaining categories were collapsed into "problem absent"

${ }^{b}$ Response category "never" was defined as "problem absent"; the remaining categories were collapsed into "problem present" 
Figure 1 Sampling procedure and results

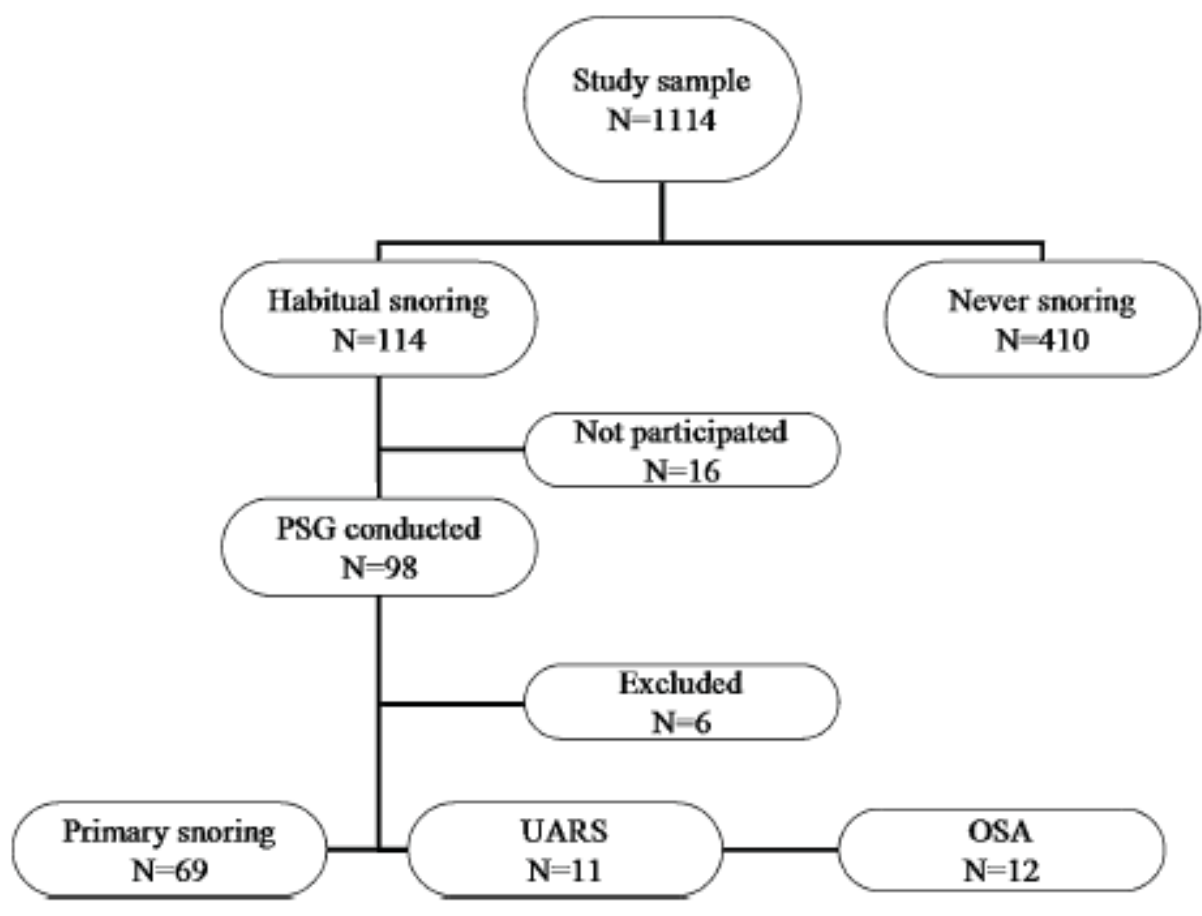

